Discrete Comput Geom 26:513-526 (2001)

DOI: $10.1007 / \mathrm{s} 00454-001-0025-\mathrm{z}$

\title{
Cyclic Product Theorems for Polygons (II) Constructions using Conic Sections
}

\author{
G. C. Shephard \\ School of Mathematics, University of East Anglia, \\ Norwich NR4 7TJ, England, U.K. \\ G.C.Shephard@uea.ac.uk
}

\begin{abstract}
If a point $U_{i}$ is chosen on each edge of a plane $n$-gon $P$, then the product of the $n$ signed ratios in which the points $U_{i}$ divide the edges of $P$ is called a cyclic product for $P$. The problem is to find geometric constructions for the $U_{i}$ such that, for every $n$-gon $P$, the cyclic product takes a fixed value. Many constructions are known which use lines or circles. Here we describe constructions that use conic sections.
\end{abstract}

\section{Introduction}

Let $P=\left[V_{0}, V_{1}, \ldots, V_{n-1}\right]$ be an $n$-gon in the Euclidean plane and let $k$ be a positive integer $(k<n)$. Also suppose that, for $i=0, \ldots, n-1$, some geometric construction leads to a point $U_{i}$ on the edge or diagonal $V_{i} V_{i+k}$ of $P$. Then the term cyclic product (for $P$ ) means an expression of the form $\prod\left(\left\|V_{i} U_{i}\right\| /\left\|U_{i} V_{i+k}\right\|\right)$. Here and throughout, when considering $n$-gons, the subscripts $i$ of the vertices $V_{i}$ are to be taken modulo $n$, and the product is from $i=0$ to $i=n-1$. The double modulus signs are used to indicate signed or directed lengths of the indicated line segments. Single modulus signs $|X Y|$ are used when unsigned lengths are required.

Familiar examples of cyclic products which take a constant value are given by Menelaus' and Ceva's theorems [5, Chapter 8]. Dozens of other examples of cyclic products which take a constant value are known (see [3], [4] and the references given there). In all of these the points $U_{i}$ are determined by straight lines and in [6] some constructions involving circles were described. In this paper we are concerned with cyclic product theorems in which the constructions use ellipses or other conic sections (conics for short). In this context there are so many possibilities that, in order to keep the treatment reasonably short, we shall only consider one type of construction that leads to a cyclic product which takes a fixed value. A typical example is shown in Fig. 3(a) for 
$n=4$. Here $F_{1}$ and $F_{2}$ are fixed points and, for $i=0,1,2,3, Q_{i}$ is the (unique) conic through $F_{1}, F_{2}$ and the three vertices $V_{i-1}, V_{i}, V_{i+1}$ of an $n$-gon $P$. If the tangent to $Q_{i}$ at $F_{1}$ meets the diagonal $V_{i-1} V_{i+1}$ in the point $U_{i}$, then

$$
\prod\left(\left\|V_{i-1} U_{i}\right\| /\left\|U_{i} V_{i+1}\right\|\right)=1 .
$$

This is the special case $k=1, r=1$ of the first assertion in Theorem 3 of Section 2. The other theorems vary in the number of fixed points, the vertices of $P$ used to define $Q$, and in the side or diagonal of $P$ that is used in the cyclic product. In Section 2 we state these theorems, followed by their proofs. Section 1 is devoted to some preliminary results and Section 3 to further discussion, comments and acknowledgements.

The polygons considered in this paper are quite general: vertices may be collinear or coincide, edges may intersect at relative interior points or overlap, etc. We make only two requirements. The first is that no three consecutive vertices of $P$ are collinear, and the second depends on the construction under consideration, namely that everything is well defined. Hence, in particular, whenever a line is defined as the join of two points then those two points are distinct; when a point is defined as the intersection of two lines, then those two lines are not parallel; when a conic is defined as passing through five points, then no three of those points are collinear. Finally, in every cyclic product that occurs none of the denominators vanish. It would, of course, be possible to specify the exact restrictions that must hold in each theorem, but as they are essentially obvious, to do so would needlessly complicate the exposition without increasing its clarity.

This paper seems to be an excellent illustration of the use of computers in pure mathematics research. Cyclic products were numerically computed for large numbers of arbitrarily chosen $n$-gons $(3 \leq n \leq 20)$ using Chipmunk and Maple $囚$ software on an iMac computer. The results that were obtained suggested the theorems of Section 2. The proofs also illustrates the power of synthetic geometric reasoning in projective grometry (in contrast to an analytic approach).

\section{Preliminary Results}

Here we review some properties of a pentagon inscribed in a conic which seem to be of interest, and which we shall require in the following two sections.

Firstly suppose that $P=\left[X_{0}, X_{1}, \ldots, X_{4}\right]$ is a pentagon inscribed in a circle $Q$. If the tangent to $Q$ at $X_{i}$ meets the edge or diagonal $X_{j} X_{k}$ in $U_{i}$ then we write $\left(X_{i} ; Q ; X_{j}, X_{k}\right)$ for the signed quotient $\left\|X_{j} U_{i}\right\| /\left\|U_{i} X_{k}\right\|$, and notice that

$$
\left(X_{i} ; Q ; X_{j}, X_{k}\right)\left(X_{i} ; Q ; X_{k}, X_{j}\right)=1 .
$$

It is convenient to introduce symbols for the following five products:

$$
\begin{aligned}
{[p, q, r]:=} & \left(X_{p} ; Q ; X_{q}, X_{r}\right)\left(X_{q} ; Q ; X_{r}, X_{p}\right)\left(X_{r} ; Q ; X_{p}, X_{q}\right), \\
{[p, q, r, s]:=} & \left(X_{p} ; Q ; X_{q}, X_{s}\right)\left(X_{q} ; Q ; X_{r}, X_{p}\right)\left(X_{r} ; Q ; X_{s}, X_{q}\right) \\
& \times\left(X_{s} ; Q ; X_{p}, X_{r}\right),
\end{aligned}
$$




$$
\begin{aligned}
{[p, q, r, s, t]:=} & \left(X_{p} ; Q ; X_{q}, X_{t}\right)\left(X_{q} ; Q ; X_{r}, X_{p}\right)\left(X_{r} ; Q ; X_{s}, X_{q}\right) \\
& \times\left(X_{s} ; Q ; X_{t}, X_{r}\right)\left(X_{t} ; Q ; X_{p}, X_{s}\right) \\
{[p ; q, r, s]:=} & \left(X_{p} ; Q ; X_{q}, X_{r}\right)\left(X_{p} ; Q ; X_{r}, X_{s}\right)\left(X_{p} ; Q ; X_{s}, X_{q}\right)
\end{aligned}
$$

and

$$
\begin{aligned}
{[p ; q, r, s, t]:=} & \left(X_{p} ; Q ; X_{q}, X_{r}\right)\left(X_{p} ; Q ; X_{r}, X_{s}\right)\left(X_{p} ; Q ; X_{s}, X_{t}\right) \\
& \times\left(X_{p} ; Q ; X_{t}, X_{q}\right),
\end{aligned}
$$

where $(p, q, r, s, t)$ is a permutation of $(0,1,2,3,4)$. The following identities are known:

$$
\begin{array}{rlrl}
10: & & {[p, q, r]} & =-1, \\
15: & & {[p, q, r, s]} & =1, \\
12: & {[p, q, r, s, t]} & =-1, \\
20: & {[p ; q, r, s]} & =-1
\end{array}
$$

and

$$
15: \quad[p ; q, r, s, t]=1,
$$

where, if we do not distinguish trivially equalities (for example $[p, q, r]=1 /[p, r, q]$ which follows from (1)) then the integer on the extreme left of each line is the number of distinct relations of the given type. Relations (7), (8) and (9) follow from Wu's theorem [Theorem 2.2 of 6], while relations (10) and (11) follow from Menelaus' theorem for a triangle or quadrangle with the tangent to $Q$ at $X_{p}$ as transversal. (Identities (7) and (10) are illustrated in Figs. 1 and 2 except that, as to be explained shortly, in these diagrams $Q$ is shown as an ellipse and not as a circle.)

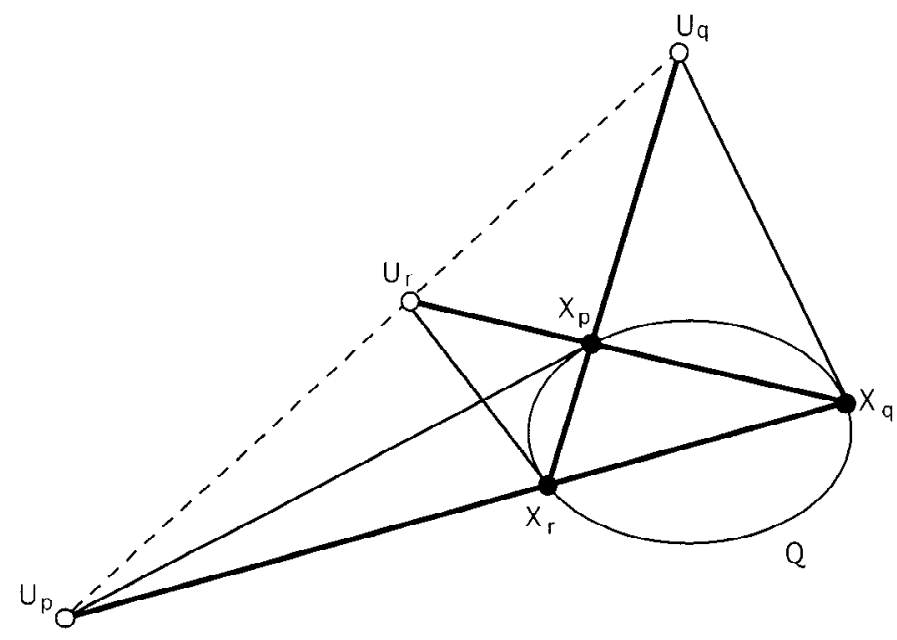

Fig. 1. An illustration of the identity $[p, q, r]=-1$ for the ellipse $Q$. 


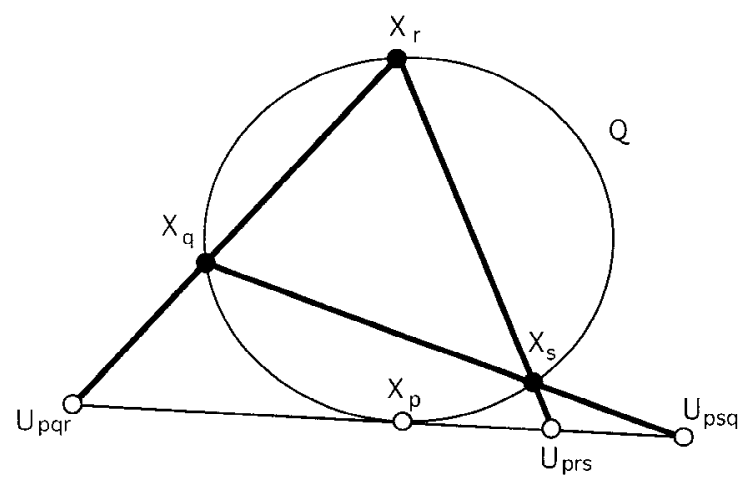

Fig. 2. An illustration of the identity $[p ; q, r, s]=-1$ for the ellipse $Q$.

Clearly the expressions (2)-(6) are not independent and the following identities hold:

$$
\begin{gathered}
{[p, q, r, s]=[p, q, r][p, r, s][p ; s, r, q][r ; s, q, p],} \\
{[p, q, r, s, t][p ; q, r, s, t][r ; s, p, q][s ; t, p, r]=[p, q, r][p, r, s][p, s, t],} \\
{[p ; q, r, s, t]=[p ; q, r, s][p ; s, t, q]=[p ; r, s, t][p ; t, q, r],} \\
{[p ; q, r, s][p ; s, t, q]=[p ; t, q, r][p ; r, s, t]}
\end{gathered}
$$

and

$$
[p ; q, r, s][r ; s, p, q][q, r, s][s, p, q]=[q ; r, s, p][s ; p, q, r][r, s, p][p, q, r] .
$$

All these identities are easily established by substituting the values given in (2)-(6).

The products on the right sides of (2)-(6) are what Howard Eves calls $h$-expressions. These are products of quotients of directed lengths of line segments such that:

(1) Each segment indicated by a term in the numerator lies on the same line as a line segment indicated by a term in the denominator, and vice versa.

(2) If each term $\|X Y\|$ is replaced by the symbolic product $x y$, then complete cancellation takes place.

By Eves' remarkable theorem [1, Theorem 6.1.9], every $h$-expression is a projective invariant, so all the above identities remain true if we apply to the diagram any (nonsingular) projective transformation. Such a transformation can be found which transforms the circle $Q$ into any chosen conic. Thus if $X_{0}, \ldots, X_{4}$ are any five points, no three of which are collinear, and $Q$ is the (unique) non-singular conic through these five points, then, with the corresponding definitions, relations (7)-(11) will hold.

\section{The Main Theorems}

We extend the notation of the previous section in the obvious manner. $(A ; Q ; B, C)$ means the signed quotient $\|B U\| /\|U C\|$ where $U$ is the point at which the tangent to 
$Q$ at $A$ meets the side or diagonal $B C$ of the polygon $P$. Here, however, $A, B, C$ are either fixed points or vertices of the $n$-gon $P=\left[V_{0}, \ldots, V_{n-1}\right]$. These points, as well as the conic $Q$, may depend on a parameter $i$ (which appears in the subscripts) and we write $\prod(A ; Q ; B, C)$ for the product of the quotients $\|B U\| /\|U C\|$ from $i=0$ to $i=n-1$.

In the statement of the following theorems, parameters $r, s, t$ may occur in the subscripts of the vertices. The given restrictions on their values ensures that the subscripts (and therefore the vertices themselves), that appear in the cyclic product symbol, are distinct. These restrictions are stated in terms of congruences modulo $n$.

A cyclic product relation for an $n$-gon is said to be primitive if it is not a consequence of a corresponding relation for some submultiple of $n$. For example, the relation $\prod\left(F ; Q_{i} ; V_{i-2}, V_{i+2}\right)=1$ for a decagon (Theorem 3 with $n=10$ and $\left.r=2\right)$ is not primitive since it follows from $\prod\left(F ; Q_{i} ; V_{i-1}, V_{i+1}\right)=-1$ for a pentagon (Theorem 3 with $n=5$ and $r=1$ ). Throughout the following theorems we only list primitive relations.

Theorem 1. For $n \geq 5$ let $Q_{i}$ be the conic through the five vertices $V_{i-r-s}, V_{i-r}, V_{i}$, $V_{i+r}$ and $V_{i+r+s}$ of $P$, where $r$ and $s$ are positive integers satisfying $r+s<n / 2$ :

(a) If $n=5$, so $r \equiv s \equiv 1$, then

$$
\prod\left(V_{i} ; Q_{i} ; V_{i-1}, V_{i+1}\right)=\prod\left(V_{i} ; Q_{i} ; V_{i-2}, V_{i+2}\right)=-1 .
$$

(b) If $n=7$ then

$(r \equiv 1, s \equiv 1)$

$$
\prod\left(V_{i-1} ; Q_{i} ; V_{i-2}, V_{i}\right)=\prod\left(V_{i+1} ; Q_{i} ; V_{i}, V_{i+2}\right)=-1,
$$

$(r \equiv 1, s \equiv 2)$

$$
\prod\left(V_{i-3} ; Q_{i} ; V_{i}, V_{i+1}\right)=\prod\left(V_{i+3} ; Q_{i} ; V_{i-1}, V_{i}\right)=-1,
$$

$(r \equiv 2, s \equiv 1)$

$$
\prod\left(V_{i-2} ; Q_{i} ; V_{i}, V_{i+3}\right)=\prod\left(V_{i+2} ; Q_{i} ; V_{i-3}, V_{i}\right)=-1 .
$$

Theorem 2. For $n \geq 4$, let $Q_{i}$ be the conic through a fixed point $F$, and the vertices $V_{i-s}, V_{i}, V_{i+r}$ and $V_{i+r+s}$ of $P$, where $r$ and $s$ are positive integers satisfying $r+2 s<n$. Then:

(a) For $n=4$, so $r \equiv s \equiv 1$,

$$
\prod\left(F ; Q_{i} ; V_{i}, V_{i+t}\right)=1 \quad \text { where } t \equiv 1,2 \text { or } 3,
$$

and

$$
\prod\left(V_{i} ; Q_{i} ; V_{i-1}, V_{i+1}\right]=1 .
$$


(b) For all $n>4$,

$$
\begin{gathered}
\prod\left(F ; Q_{i} ; V_{i}, V_{i+r}\right)=(-1)^{n} \quad \text { if } \quad 3 s+2 r \equiv 0 \quad \text { or } \quad 3 s+r \equiv 0, \\
\prod\left(F ; Q_{i} ; V_{i-s}, V_{i+r+s}\right)=(-1)^{n} \quad \text { if } \quad s+2 r \equiv 0 \quad \text { or } \quad s \equiv r .
\end{gathered}
$$

(c) For even $n>4$,

$$
\begin{aligned}
& \prod\left(F ; Q_{i} ; V_{i-s}, V_{i}\right) \\
& \quad=\prod\left(F ; Q_{i} ; V_{i+r}, V_{i+r+s}\right)=1 \quad \text { if } s \equiv n / 2, \\
& \begin{aligned}
\prod\left(F ; Q_{i} ;\right. & \left.V_{i-s}, V_{i+r}\right) \\
& =\prod\left(F ; Q_{i} ; V_{i}, V_{i+r+s}\right)=1
\end{aligned}
\end{aligned}
$$

Theorem 3. Let $Q_{i}$ be the conic through the fixed points $F_{1}, F_{2}$ and the vertices $V_{i-r}, V_{i}$ and $V_{i+r}$ of $P$, where $r$ is a positive integer satisfying $r<n / 2$. Then for all $n \geq 3$ and $k=1$ or 2 ,

$$
\prod\left(F_{k} ; Q_{i} ; V_{i-r}, V_{i+r}\right)=\prod\left(V_{i} ; Q_{i} ; V_{i-r}, V_{i+r}\right)=(-1)^{n} .
$$

(See Fig. 3.)

Theorem 4. Let $Q_{i}$ be the conic through the fixed points $F_{1}, F_{2}, F_{3}$ and the vertices $V_{i}, V_{i+r}$ of $P$, where $r<n$. Then for all $n \geq 3$ and $k=1,2$ or 3 ,

$$
\prod\left(F_{k} ; Q_{i} ; V_{i}, V_{i+r}\right)=(-1)^{n} .
$$

If $m$ is the number of fixed points, then Theorems 1, 2, 3 and 4 correspond to $m=$ $0,1,2$ and 3 respectively. For $m=5$ the cyclic products are trivial since they contain only one term; these are dealt with in Section 1. The absence of any cyclic product relations in the case $m=4$ is a consequence of the following theorem:

Theorem 5. There are no cyclic product relations for n-gons of the form

$$
\prod\left(A ; Q_{i} ; B, C\right)=\text { constant }
$$

(where the constant may depend on $n$, but is independent of the $n$-gon $P$ ) when either $B$ or $C$ is a fixed point, and the other symbols represent either fixed points or vertices of $P$.

We believe that Theorems $1-4$ cover all primitive cyclic product relations which take a fixed value for all $n$-gons $P$. This belief is based on numerical checking for arbitrarily chosen $n$-gons for various values of $n$ up to 20 . In particular, numerical evidence indicates that there are no primitive cyclic products of the kind considered in Theorem 1 for $n=6$ or $n \geq 8$, though, of course, an infinity of non-primitive such relations exist.

The following lemma is fundamental. 


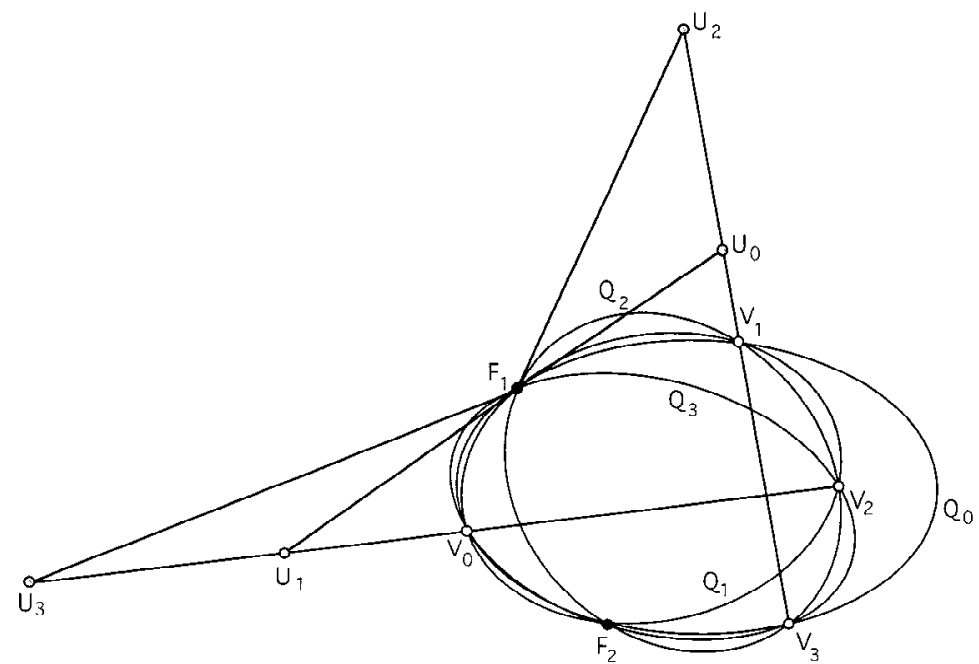

(a)

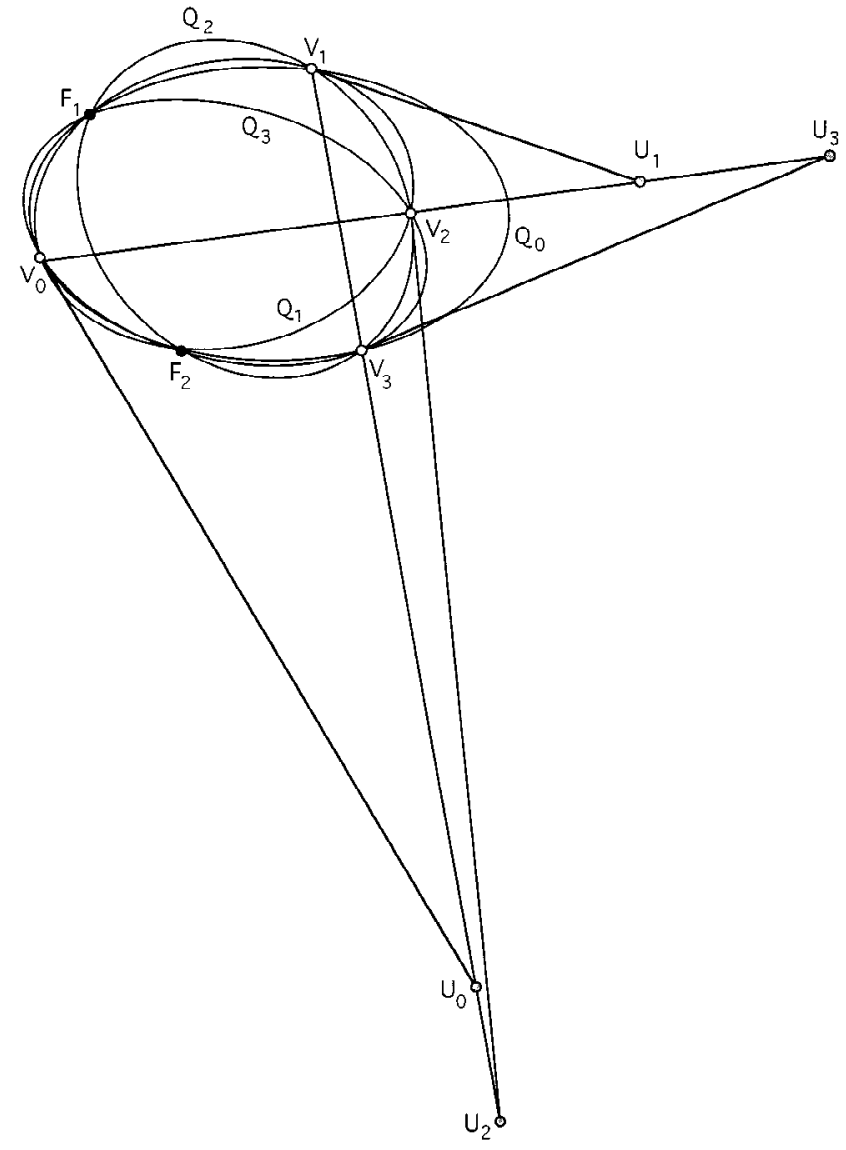

(b)

Fig. 3. An illustration of Theorem 3. 


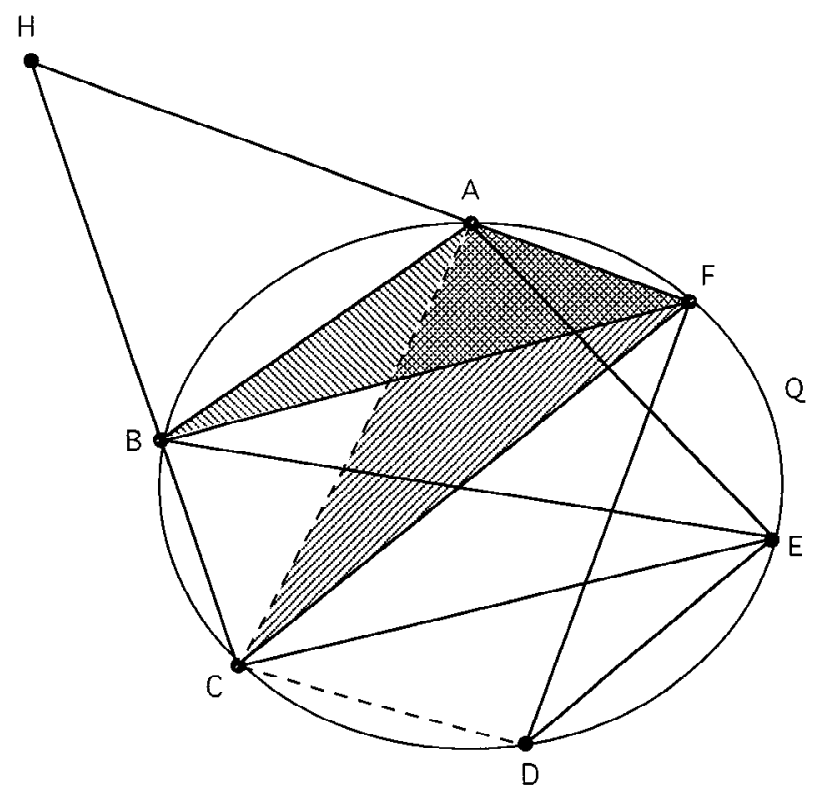

(a)

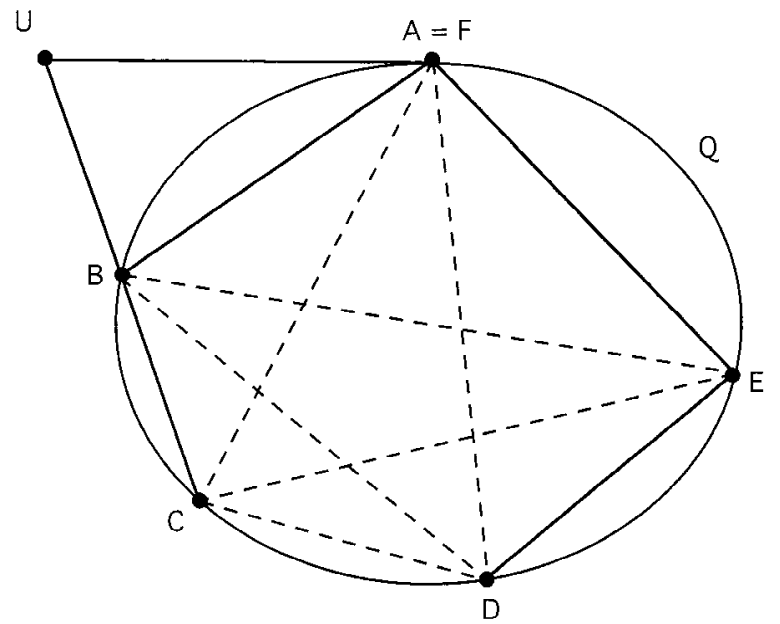

(b)

Fig. 4. The proof of the lemma.

Lemma 1. Let $A, B, C, D, E$ be five distinct points on a conic $Q$, and suppose the tangent to $Q$ at $A$ meets the line $B C$ in $U$. Then

$$
(A ; Q ; B, C)=-\frac{\|A E B\|}{\|A E C\|} \cdot \frac{\|D E C\|}{\|D E B\|} \cdot \frac{\|A D B\|}{\|A D C\|},
$$

where the six terms on the right are the signed areas of the indicated triangles. (See Fig. 4(b).) 
We recall that the area of a triangle is positive if its vertices are listed in a counterclockwise direction, and negative if listed in a clockwise direction.

Proof. Consider the pencil of lines $E(A, D, B, C)$ joining $E$ to the other four points. If $\theta_{1}=\angle A E B, \theta_{2}=\angle A E C, \theta_{3}=\angle D E B, \theta_{4}=\angle D E C$, where each of these angles lies between $-\pi$ and $\pi$ radians, then the cross ratio of this pencil is

$$
\begin{aligned}
\operatorname{cr}(E(A, D ; B, C)) & =\frac{\sin \theta_{1}}{\sin \theta_{2}} / \frac{\sin \theta_{3}}{\sin \theta_{4}} \\
& =\frac{\frac{1}{2}|E A| \cdot|E B| \cdot \sin \theta_{1}}{\frac{1}{2}|E A| \cdot|E C| \cdot \sin \theta_{2}} / \frac{\frac{1}{2}|E D| \cdot|E B| \cdot \sin \theta_{3}}{\frac{1}{2}|E D| \cdot|E C| \cdot \sin \theta_{4}} \\
& =\frac{\|A E B\|}{\|A E C\|} / \frac{\|D E B\|}{\|D E C\|} .
\end{aligned}
$$

Now let $F$ be any other point on $Q$ (see Fig. 4(a)). As above,

$$
\operatorname{cr}(F(A, D ; B, C))=\frac{\|A F B\|}{\|A F C\|} / \frac{\|D F B\|}{\|D F C\|} .
$$

By a fundamental property of conics [1, Theorem 6.3.1], these two cross ratios are equal. Hence, from (22) and (23), after rearranging,

$$
\frac{\|A F B\|}{\|A F C\|}=\frac{\|D F B\|}{\|D F C\|} \cdot \frac{\|A E B\|}{\|A E C\|} \cdot \frac{\|D E C\|}{\|D E B\|} .
$$

If $A F$ meets $B C$ in $H$ then by the area principle (see [3]) since the triangles [AFB] and $[A F C]$ have the same base $[A F]$ and their heights are proportional to $|B H|$ and $|C H|$, taking account of signs, the left side of (24) is equal to $\|B H\| /\|C H\|$.

Consider the limit as $F \rightarrow A$ on $Q$ (Fig. 4(b)). The chord $A F$ tends to the tangent to $Q$ at $A$ and so the point $H$ tends to $U$. In the limit we get

$$
\frac{\|B U\|}{\|C U\|}=\frac{\|D A B\|}{\|D A C\|} \cdot \frac{\|A E B\|}{\|A E C\|} \cdot \frac{\|D E C\|}{\|D E B\|}
$$

which, since $(A ; Q ; B, C)=\|B U\| /\|U C\|$ is clearly equivalent to (21).

The proofs of many parts of Theorems 1-4 are very similar; we use the lemma with suitable substitutions for the points $A, B, C, D$ and $E$, take the product from $i=0$ to $i=n-1$ and then rearrange the terms. The required equalities follow immediately. To avoid tedious repetitions we give full details in one case only, namely in the proof of part of Theorem 2. In other cases we only state the required substitution and the form of the rearranged product.

To prove (16) of Theorem 2(b) we substitute $F, V_{i}, V_{i+r}, V_{i-s}, V_{i+r+s}$ for $A, B, C$, $D, E$ respectively in the lemma, yielding

$$
\left(F ; Q_{i} ; V_{i}, V_{i+r}\right)=-\frac{\left\|F V_{i+r+s} V_{i}\right\|}{\left\|F V_{i+r+s} V_{i+r}\right\|} \cdot \frac{\left\|V_{i-s} V_{i+r+s} V_{i+r}\right\|}{\left\|V_{i-s} V_{i+r+s} V_{i}\right\|} \cdot \frac{\left\|F V_{i-s} V_{i}\right\|}{\left\|F V_{i-s} V_{i+r}\right\|} .
$$


Taking the product from $i=0$ to $n-1$ we obtain

$$
\begin{aligned}
& \prod\left(F ; Q_{i} ; V_{i}, V_{i+r}\right) \\
& =(-)^{n} \prod \frac{\left\|F V_{i+r+s} V_{i}\right\|}{\left\|F V_{i+r+s} V_{i+r}\right\|} \cdot \prod \frac{\left\|V_{i-s} V_{i+r+s} V_{i+r}\right\|}{\left\|V_{i-s} V_{i+r+s} V_{i}\right\|} \cdot \prod \frac{\left\|F V_{i-s} V_{i}\right\|}{\left\|F V_{i-s} V_{i+r}\right\|} \\
& =(-)^{n} \prod \frac{\left\|F V_{i+r+s} V_{i}\right\|}{\left\|F V_{i-s} V_{i+r}\right\|} \cdot \prod \frac{\left\|F V_{i-s} V_{i}\right\|}{\left\|F V_{i+r+s} V_{i+r}\right\|} \cdot \prod \frac{\left\|V_{i-s} V_{i+r+s} V_{i+r}\right\|}{\left\|V_{i-s} V_{i+r+s} V_{i}\right\|}
\end{aligned}
$$

upon rearranging. Now each of the first two products on the right is equal to $(-1)^{n}$. To see this, substitute $i \rightarrow i-s$ and $i \rightarrow i+r+s$ in the numerators of the two products, respectively, observing that the triangles in the numerator and denominator of each fraction have opposite orientations. The third product has value 1 as we can see by substituting either $i \rightarrow i-r-s$ (noting that $i-r-2 s \equiv i+r+s$ if $3 s+2 r \equiv 0$ ) or $i \rightarrow i+s$ (noting that $i+r+2 s \equiv i-s$, if $3 s+r \equiv 0$ ) in the numerators. This proves (16).

Proof of Theorem 1. (a) The two assertions in (12) are equivalent to

$$
[0,1,2,3,4]=[0,2,4,1,3]=-1
$$

which were proved in the previous section, applied to the pentagon $\left[V_{0}, V_{1}, V_{2}, V_{3}, V_{4}\right]$.

(b) All six assertions are proved in a similar manner; we only indicate the proof of the first equality in (13). Substitute $V_{i-3}, V_{i}, V_{i+1}, V_{i+3}, V_{i-1}$ for $A, B, C, D, E$ respectively in the lemma. Taking the product from $i=0$ to $i=6$ and rearranging,

$$
\begin{aligned}
\prod\left(V_{i-3} ;\right. & \left.Q_{i} ; V_{i}, V_{i+r}\right) \\
= & -\prod \frac{\left\|V_{i-3} V_{i-1} V_{i}\right\|}{\left\|V_{i-3} V_{i+3} V_{i+1}\right\|} \cdot \prod \frac{\left\|V_{i+3} V_{i-1} V_{i+1}\right\|}{\left\|V_{i-3} V_{i-1} V_{i+1}\right\|} \cdot \prod \frac{\left\|V_{i-3} V_{i+3} V_{i}\right\|}{\left\|V_{i+3} V_{i-1} V_{i}\right\|}=-1 .
\end{aligned}
$$

Proof of Theorem 2. (a) Here, since $n=4$ and there is one fixed point, $Q_{i}$ is fixed, that is, independent of $i$. Hence (14) follows from (11) if $t=1$ or 3 , and from (1) if $t=2$. Similarly (15) follows from (8).

(b) The first assertion (16) is proved above. For the second, substitute $F, V_{i-s}, V_{i+r+s}$, $V_{i}, V_{i+r}$ for $A, B, C, D, E$ respectively in the lemma. Taking the product from $i=0$ to $n-1$ and rearranging we obtain

$$
\begin{aligned}
& \prod\left(F ; Q_{i} ; V_{i-s}, V_{i+r+s}\right) \\
& \quad=(-)^{n} \prod \frac{\left\|F V_{i+r} V_{i-s}\right\|}{\left\|F V_{i} V_{i+r+s}\right\|} \cdot \prod \frac{\left\|F V_{i} V_{i-s}\right\|}{\left\|F V_{i+r} V_{i+r+s}\right\|} \cdot \prod \frac{\left\|V_{i} V_{i+r} V_{i+r+s}\right\|}{\left\|V_{i} V_{i+r} V_{i-s}\right\|}=(-1)^{n} .
\end{aligned}
$$

This proves (17).

(c) Substitute $F, V_{i-s}, V_{i}, V_{i+r}, V_{i+r+s}$ for $A, B, C, D, E$ respectively in the lemma. Taking products and rearranging,

$$
\begin{aligned}
\prod\left(F ; Q_{i} ;\right. & \left.V_{i-s}, V_{i}\right) \\
& =\prod \frac{\left\|F V_{i+r+s} V_{i-s}\right\|}{\left\|F V_{i+r} V_{i}\right\|} \cdot \prod \frac{\left\|F V_{i+r} V_{i-s}\right\|}{\left\|F V_{i+r+s} V_{i}\right\|} \cdot \prod \frac{\left\|V_{i+r} V_{i+r+s} V_{i}\right\|}{\left\|V_{i+r} V_{i+r+s} V_{i-s}\right\|}=1 .
\end{aligned}
$$


For the second equality, substitute $F, V_{i+r}, V_{i+r+s}, V_{i-s}, V_{i}$, for $A, B, C, D, E$ respectively in the lemma. Taking products and rearranging,

$$
\begin{aligned}
\prod\left(F ; Q_{i} ; V_{i+r}, V_{i+r+s}\right) \\
\quad=\prod \frac{\left\|F V_{i} V_{i+r}\right\|}{\left\|F V_{i-s} V_{i+r+s}\right\|} \cdot \prod \frac{\left\|F V_{i-s} V_{i+r}\right\|}{\left\|F V_{i} V_{i+r+s}\right\|} \cdot \prod \frac{\left\|V_{i-s} V_{i} V_{i+r+s}\right\|}{\left\|V_{i-s} V_{i} V_{i+r}\right\|}=1 .
\end{aligned}
$$

For the third equality substitute $F, V_{i-s}, V_{i+r}, V_{i}, V_{i+r+s}$ for $A, B, C, D, E$ respectively in the lemma. Taking products and rearranging,

$$
\begin{aligned}
\prod\left(F ; Q_{i} ; V_{i-s}, V_{i+r}\right) \\
\quad=\prod \frac{\left\|F V_{i+r+s} V_{i-s}\right\|}{\left\|F V_{i} V_{i+r}\right\|} \cdot \prod \frac{\left\|F V_{i} V_{i-s}\right\|}{\left\|F V_{i+r+s} V_{i+r}\right\|} \cdot \prod \frac{\left\|V_{i} V_{i+r+s} V_{i+r}\right\|}{\left\|V_{i} V_{i+r+s} V_{i-s}\right\|}=1 .
\end{aligned}
$$

For the fourth and final equality, substitute $F, V_{i}, V_{i+r+s}, V_{i-s}, V_{i+r}$ for $A, B, C, D, E$ respectively in the lemma. Taking products and rearranging,

$$
\begin{aligned}
& \prod\left(F ; Q_{i} ; V_{i}, V_{i+r+s}\right) \\
& \quad=\prod \frac{\left\|F V_{i+r} V_{i}\right\|}{\left\|F V_{i-s} V_{i+r+s}\right\|} \cdot \prod \frac{\left\|F V_{i-s} V_{i}\right\|}{\left\|F V_{i+r} V_{i+r+s}\right\|} \cdot \prod \frac{\left\|V_{i-s} V_{i+r} V_{i+r+s}\right\|}{\left\|V_{i-s} V_{i+r} V_{i}\right\|}=1 .
\end{aligned}
$$

This proves (18) and concludes the proof of Theorem 2.

Proof of Theorem 3. Consider the case $k=1$. Substitute $F_{1}, V_{i-r}, V_{i+r}, F_{2}, V_{i}$ for $A, B, C, D, E$ respectively in the lemma. Taking products,

$$
\begin{aligned}
\prod\left(F_{1} ; Q_{i} ; V_{i-r}, V_{i+r}\right) & =(-)^{n} \prod \frac{\left\|F_{1} V_{i} V_{i-r}\right\|}{\left\|F_{1} V_{i} V_{i+r}\right\|} \cdot \prod \frac{\left\|F_{2} V_{i} V_{i+r}\right\|}{\left\|F_{2} V_{i} V_{i-r}\right\|} \cdot \prod \frac{\left\|F_{1} F_{2} V_{i-r}\right\|}{\left\|F_{1} F_{2} V_{i+r}\right\|} \\
& =(-1)^{n} .
\end{aligned}
$$

For the second assertion, substitute $V_{i}, V_{i-r}, V_{i+r}, F_{1}, F_{2}$ for $A, B, C, D, E$ respectively in the lemma. Taking products,

$$
\begin{aligned}
\prod\left(V_{i} ; Q_{i} ; V_{i-r}, V_{i+r}\right) & =(-)^{n} \prod \frac{\left\|V_{i} F_{2} V_{i-r}\right\|}{\left\|V_{i} F_{2} V_{i+r}\right\|} \cdot \prod \frac{\left\|F_{1} F_{2} V_{i+r}\right\|}{\left\|F_{1} F_{2} V_{i-r}\right\|} \cdot \prod \frac{\left\|V_{i} F_{1} V_{i-r}\right\|}{\left\|V_{i} F_{1} V_{i+r}\right\|} \\
& =(-1)^{n} .
\end{aligned}
$$

This proves (19) and concludes the proof of Theorem 3.

Proof of Theorem 4. Substitute $F_{1}, V_{i}, V_{i+r}, F_{2}, F_{3}$ for $A, B, C, D, E$ respectively in the lemma. Taking products,

$$
\begin{aligned}
\prod\left(F_{1} ; Q_{i} ; V_{i}, V_{i+r}\right) & =(-)^{n} \prod \frac{\left\|F_{1} F_{3} V_{i}\right\|}{\left\|F_{1} F_{3} V_{i+r}\right\|} \cdot \prod \frac{\left\|F_{2} F_{3} V_{i+r}\right\|}{\left\|F_{2} F_{3} V_{i}\right\|} \cdot \prod \frac{\left\|F_{1} F_{2} V_{i}\right\|}{\left\|F_{1} F_{2} V_{i+r}\right\|} \\
& =(-1)^{n} .
\end{aligned}
$$

This establishes equality (20). 
Proof of Theorem 5. We need to show that if $B$ is a fixed point, then $\prod\left(A ; Q_{i} ; B, C\right)$ is not a constant, that is, it does not take the same value for all $n$-gons $P$. Choose a value of $n \geq 3$. Let $Q$ be a circle of diameter $d$, and suppose that all the fixed points and the vertices of the $n$-gon $P$ lie on $Q$. Then $Q_{i}=Q$ for all $i=0, \ldots, n-1$. Let $B X$ be a diameter of $Q$, and suppose that all the points (except $B$ ) lie near to $X$. Whether $A$ and $C$ are fixed points or not, by Lemma 2 of [6], we see

$$
(A ; Q ; B, C)=-|A B|^{2} /|A C|^{2}
$$

and $\prod\left(A ; Q_{i} ; B, C\right)$ is a product of such terms. By selecting $P$ so that its vertices, and all the fixed points other than $B$, lie close to $X$, the term $|A C|^{2}$ may be made as small as we wish, whereas $|A B|^{2}$ is close to $d^{2}$. Hence we can make $\prod\left(A ; Q_{i} ; B, C\right)$ as large as we wish, and therefore it cannot take a constant value.

\section{Other Relations}

So far we have restricted attention to the consideration of cyclic products which take a constant value for all $n$-gons $P$. In addition there are numerous relations between the values of two, three, ..., cyclic products. So many, in fact, that it is impractical to give an exhaustive treatment, and we restrict ourselves to some suggestions as to how they may be constructed, and a few examples.

Each of the relations (7)-(11) of Section 1 gives rise to many relations. Consider the following. Take five points, each of which may be either a fixed point or any vertex of a given $n$-gon $P$. For example, suppose there are $m=2$ fixed points $F_{1}$ and $F_{2}$, and the chosen vertices of $P$ are $V_{i-r}, V_{i}, V_{i+s}$, where $r$ and $s$ are positive integers such that $r+s<n$. Let $Q_{i}$ be the unique conic through these five points. Notice that, unlike Theorem 3, initially we do not require $r=s$. Then, by (2) and (7),

$$
\left.\left(V_{i-r} ; Q_{i} ; V_{i}, V_{i+s}\right)\left(V_{i} ; Q_{i} ; V_{i+s}, V_{i-r}\right)\left(V_{i+s} ; Q_{i} ; V_{i-r}, V_{i}\right)\right)=-1 .
$$

Taking the product from $i=0$ to $i=n-1$, we obtain the relation

$$
\prod\left(V_{i-r} ; Q_{i} ; V_{i}, V_{i+s}\right) \prod\left(V_{i} ; Q_{i} ; V_{i+s}, V_{i-r}\right) \prod\left(V_{i+s} ; Q_{i} ; V_{i-r}, V_{i}\right)=(-1)^{n}
$$

connecting three cyclic products for $P$. Further, if $r=s$, we know from Theorem 3 that $\prod\left(V_{i} ; Q_{i} ; V_{i+r}, V_{i-r}\right)=(-1)^{n}$, and hence we obtain the identity

$$
\prod\left(V_{i-r} ; Q_{i} ; V_{i}, V_{i+r}\right) \prod\left(V_{i+r} ; Q_{i} ; V_{i-r}, V_{i}\right)=1,
$$

which is true for all $n$-gons, and is easily verified numerically.

Another situation in which the product of two cyclic products takes a fixed value arises from a process analogous to "asymmetrisation" described in [6]. For example, we know that, under the conditions of Theorem 3 (equation (20)),

$$
\prod\left(V_{i} ; Q_{i} ; V_{i-r}, V_{i+r}\right)=(-1)^{n},
$$

where $Q_{i}$ is the conic through the fixed points $F_{1}, F_{2}$ and the three vertices $V_{i}, V_{i-r}, V_{i+r}$ of $P$. Consider

$$
\prod\left(V_{i} ; Q_{i} ; V_{i-r}, V_{i+s}\right) \prod\left(V_{i} ; Q_{i}^{\prime} ; V_{i-s}, V_{i+r}\right)
$$


where $r$ and $s$ are positive integers such that $r+s<n, Q_{i}$ is the conic through the fixed points $F_{1}, F_{2}$ and the vertices $V_{i}, V_{i-r}, V_{i+s}$ of $P$, and $Q_{i}^{\prime}$ is the conic through the same fixed points and the vertices $V_{i}, V_{i+r}, V_{i-s}$ of $P$.

Then the product (25) takes the fixed value 1 for all $n \geq 5$, as we can see as follows. Applying the lemma in the appropriate fashion,

$$
\left(V_{i} ; Q_{i} ; V_{i-r}, V_{i+s}\right)=-\frac{\left\|V_{i} F_{2} V_{i-r}\right\|}{\left\|V_{i} F_{2} V_{i+s}\right\|} \cdot \frac{\left\|F_{1} F_{2} V_{i+s}\right\|}{\left\|F_{1} F_{2} V_{i-r}\right\|} \cdot \frac{\left\|V_{i} F_{1} V_{i-r}\right\|}{\left\|V_{i} F_{1} V_{i+s}\right\|}
$$

and

$$
\left(V_{i} ; Q_{i}^{\prime} ; V_{i-s}, V_{i+r}\right)=-\frac{\left\|V_{i} F_{2} V_{i-s}\right\|}{\left\|V_{i} F_{2} V_{i+r}\right\|} \cdot \frac{\left\|F_{1} F_{2} V_{i+r}\right\|}{\left\|F_{1} F_{2} V_{i-s}\right\|} \cdot \frac{\left\|V_{i} F_{1} V_{i-s}\right\|}{\left\|V_{i} F_{1} V_{i+r}\right\|}
$$

Now take the products of each of these expressions from $i=0$ to $i=n-1$. Clearly all the terms on the right sides cancel to yield the value 1 .

Throughout we have only been concerned with cyclic products that arise in one particular way, which may be regarded as the analogue of Wu's theorem for circles [6]. Nevertheless many more constructions are possible, and each leads to cyclic products whose properties have yet to be investigated. As the constructions and the relations between the cyclic products become more and more complicated, so their intuitive appeal diminishes.

However, one such construction seems worthy of mention. If a conic is inscribed in a pentagon $P=\left[V_{0}, \ldots, V_{4}\right]$ and touches each side $V_{i} V_{i+1}$ in the point $W_{i}$ (see Fig. 5), then it is easy to show that

$$
\prod\left(\left\|V_{i+1} W_{i}\right\| /\left\|W_{i} V_{i}\right\|\right)=1 .
$$

This, and a number of similar results follow from the procedure described in Section 1: Any statement about cyclic products which involves only a unique conic, can, by a projective transformation, be transformed into one in which the conic is replaced by a circle. This usually simplifies the geometry considerably.

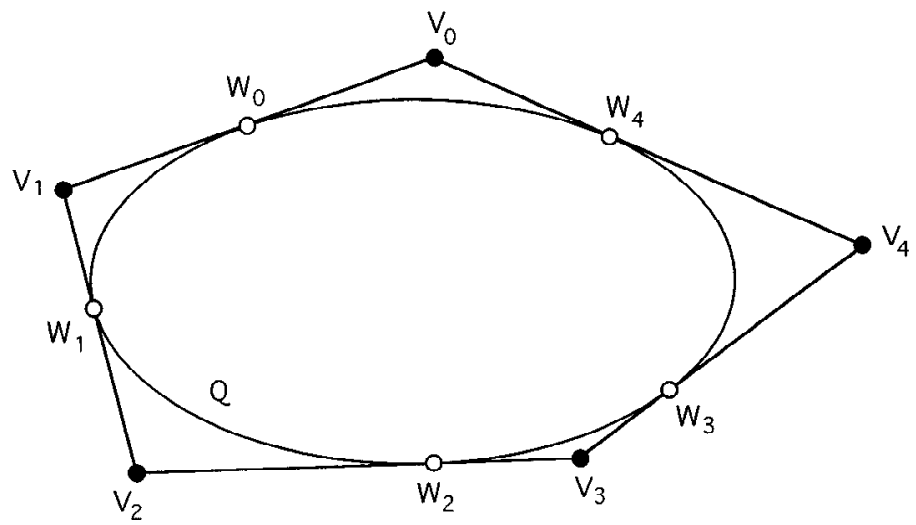

Fig. 5. If the conic $Q$ touches the sides of the pentagon $\left[V_{0}, V_{1}, V_{2}, V_{3}, V_{4}\right]$ at the points $W_{i}$ as shown, then $\prod\left(\left\|V_{i} W_{i}\right\| /\left\|W_{i} V_{i+1}\right\|\right)=1$, see Section 3 . 
Based on numerical evidence, a few of the results of this paper were conjectured by Branko Grünbaum and appear in his unpublished lecture notes [2]. The ease with which our theorems can be deduced from the lemma illustrates the fact that classical synthetic methods can, under certain circumstances, be much more powerful than, say, an analytic approach.

\section{Acknowledgement}

I am indebted to the referee for making the following suggestion: The theorems of this paper, as well as some of those in [6] and possibly many others, may be consequences of a more fundamental result, perhaps concerning general algebraic curves. Without further evidence, however, it seems impossible to even conjecture any statement of this nature.

\section{References}

1. Howard W. Eves, A Survey of Geometry, Allyn and Bacon, Boston, MA, 1972.

2. Branko Grünbaum, unpublished lecture notes on "Modern Elementary Geometry" for a course given at the University of Washington, 1997.

3. Branko Grünbaum and G. C. Shephard, Ceva, Menelaus and the area principle, Math. Mag. 68 (1995), 254-268.

4. Branko Grünbaum and G. C. Shephard, Some new transversality properties, Geom. Dedicata, 71 (1998), 179-208.

5. R. A. Johnson, Advanced Euclidean Geometry (Modern Geometry), Houghton Mifflin, 1929. (Reprint: Dover, New York, 1960.)

6. G. C. Shephard, Cyclic product theorems for polygons (I) Constructions using circles, Discrete Comput. Geom., 24 (2000), 551-571.

Received August 23, 2000, and in revised form November 27, 2000. Online publication May 4, 2001. 